\title{
Sur la Condition Nécessaire du Problème Mixte Bien Posé pour les Systémes Hyperboliques à Coefficients Variables
}

\author{
Par \\ Kunihiko KajITANI*
}

\section{\$1. Introduction}

Dans cet article, nous traiterons les systèmes hyperboliques du premier ordre à coefficients variables. Notre but est d'envisager la nécessité de la condition de Lopotinski, pour que le problème mixte pour ces systémes soit bien posé dans l'espace $L^{2}$ ou $\mathscr{E}$. Dans le cas des coefficients constants, dans [2] et [3] $\mathrm{R}$. Hersh a prouvé que la condition de Lopatinski est nécessaire et suffisante pour que le problème mixte soit bien posé dans l'espace $\mathscr{E}$.

Nous considérons le problème mixte du systéme suivant; $R_{+}^{k}=\left\{x \in R^{k}\right.$, $\left.x_{k}>0\right\}$,

$$
\left\{\begin{array}{l}
L[u(x, t)]=f(x, t), \quad t>0, \quad x \in R_{+}^{k}, \\
u(x, 0)=g(x), \quad x \in R_{+}^{k}, \\
P\left[u\left(x^{\prime}, 0, t\right)\right]=h\left(x^{\prime}, t\right), \quad t>0, \quad x^{\prime} \in R^{k-1},
\end{array}\right.
$$

où $L=\frac{\partial}{\partial t}-\sum_{i=1}^{k} A_{j}(x, t) \frac{\partial}{\partial x_{j}}-B(x, t)$ et $A_{j}(x, t), j=1, \ldots, k$, et $B(x, t)$ sont des matrices d'ordre $m$ dont les éléments sont des fonctions suffisamment régulières, et $P\left(x^{\prime}, t\right)$ une matrice à $l$ lignes et $m$ colonnes de rang $l$, d'élément suffisamment régulières. Nons supposons que $A_{k}(0,0)$ a la forme suivante,

Communicated by S. Matsuura, November 15, 1972.

* Department of Applied Mathematics and Physics, Faculty of Engineering, Kyoto University, Kyoto. 


$$
A_{k}(0,0)=\left(\begin{array}{cccccc}
a_{1} & & & & & \\
& & & & & \\
& a_{2} & & & \\
& & \ddots & & & \\
& & a_{p} & & \\
& 0 & & & a_{p+1} & \\
& & & & & \\
& & & & & a_{m}
\end{array}\right),
$$

où $a_{j}, j=1,2, \ldots, p$ sont des nombres négatifs et $a_{j}, j=p+1, \ldots, m$, sont positifs.

Nous supposons désormais que $L$ est hyperbolique, à savoir que toutes les valeurs propres de la matrice $\sum_{j=1}^{k} A_{j}(x, t) \xi_{j}$ soient réelles pour tout $\xi \in R^{k}-\{0\}$ et $(x, t) \in R^{k} \times[0, T]$, et que $A_{k}\left(x^{\prime}, 0, t\right)$ est non singulière pour tout $\left(x^{\prime}, t\right) \in R^{k-1} \times[0, T]$. On considére la matrice suivante dépendante des paramétres $\lambda$ complexes, $\operatorname{Re} \lambda>0$, et $\eta \in R^{k-1}$,

$$
M(x, t ; \lambda, i \eta)=A_{k}^{-1}(x, t)\left(\lambda-i \sum_{j=1}^{k-1} A_{j}(x, t) \eta_{j}\right) .
$$

Alors il découle de l'hyperbolicité de $L$ que pour tout $\eta \in R^{k-1}$ et $\lambda$ complexe, $\operatorname{Re} \lambda>0$, aucune des valeurs propres de $M(x, t ; \lambda, i \eta)$ n'est imaginaire pure. Donc, on a une matrice non singulière $N(x, t ; \lambda, i \eta)$, dont les éléments varient continûment en $(x, t ; \lambda, i \eta)$, telle que

$$
N^{-1} M N=\left(\begin{array}{cc}
M^{-} & * \\
0 & M^{+}
\end{array}\right),
$$

où $M^{-}(x, t ; \lambda, i \eta)$ (resp. $M^{+}(x, t ; \lambda, i \eta)$ ) est une matrice d'ordre $p$ (resp. d'ordre $m-p$ ), dont les valeurs propres ont la paritie réelle négative (resp. positive), $p$ désigne ici le nombre de la valeur propre négative de $A_{k}$. On décompose $N=\left(N^{-}, N^{+}\right)$, où $N^{-}$(resp. $\left.N^{+}\right)$est une matrice à $m$ lignes et $p$ (resp. $m-p$ ) colonnes.

Déffrmition $\mathbb{1}$.耳. On dit que $(L, P)$ satisfait à la condition de Lopatinski, si les propriétées suivantes sont vérifiées,

i) $l=p \quad$ et

ii) la $l \times l$ matrice $P\left(x^{\prime}, t\right) N^{-}\left(x^{\prime}, 0, t ; \lambda, i \eta\right)$ est non singulière pour tout $\left(x^{\prime}, t\right) \in R^{k-1} \times[0, T], \lambda$ complexe, $\operatorname{Re} \lambda>0$ et 
$\eta \in R^{k-1}$ 。

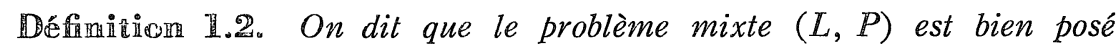
dans l'espace $L^{2}$, si pour tout $f(t) \in \mathscr{E}_{t}^{1}\left(L^{2}\right) \cap \mathscr{E}_{l}^{0}\left(H^{1}\right), g=0$ et $h=0$, il existe une solution $u(t) \in \mathscr{E}_{t}^{1}\left(L^{2}\right) \cap \mathscr{E}_{t}^{0}\left(H^{1}\right)$ vérifiant (1.1) avec $g=0$ et $h=0$ telle qu'on ait, $T>0$,

$$
\|u(t)\| \leqq \text { const. } \int_{0}^{t}\|f(s)\| d s, \text { pour } t \in(0, T) .
$$

$\|\cdot\|$ désigne ici la norme naturelle de $L^{2}\left(R_{+}^{k}\right), H^{1}=H^{1}\left(R_{+}^{k}\right)$ l'espace de Sobolev et $\mathscr{E}_{t}^{p}\left(L^{2}\right)$ (resp. $\left.\mathscr{E}_{t}^{p}\left(H^{1}\right)\right), p=0,1,2, \ldots$, ast constitué des foncitions $p$ fois continûment différentiables dans l'espace $L^{2}$ (resp. $H^{1}$ ).

Nous allons maintenant énoncer notre résultat.

Thécrème $\mathbb{1} . \mathbb{1}$. On suppose que le problème mixte $(L, P)$ est bien posé dans l'espace $L^{2}$. Alors il faut que la matrice $P\left(x^{\prime}, t\right)$ satisfasse la condition de Lopatinski.

On va établir ce théorème dans le paragraphe 2 . Nous devons signaler que récemment $\mathrm{R}$. Sakamoto, $[7]$, a dérivé la condition nécessaire, pour que les équations hyperboliques d'ordre supérieur soient bien posées dans l'espace $L^{2}$. Elle analyse dans [7] des éléments de la matrice de Lopatinski. De plus, nous remarquons que dans [1] R. Agemi et T. Shirota envisagent la condition nécessaire pour que les équations hyperboliques d'ordre supérieur soient bien posées dans l'espace $L^{2}$.

Dans le paragraphe 4 , nous allons envisager la condition nécessaire, pour que la solution du problème mixte (1.1) avec $g=0$ vérifie, au lieu de (1.2) l'inégalite suivante, pour tout $\mu>\mu_{0}>0$,

$$
\begin{aligned}
& \mu \int_{0}^{T}\left\|e^{-\mu t} u(t)\right\|^{2} d t+\int_{0}^{T}<e^{-\mu t} u(t)>^{2} d t \\
& \leqq \text { const. }\left\{\frac{1}{\mu} \int_{0}^{T}\left\|e^{-\mu t} f(t)\right\|^{2} d t+\int_{0}^{T}<e^{-\mu t} h(t)>^{2} d t\right\},
\end{aligned}
$$

où la constante ne dépend pas de $\mu_{0} \quad\langle\bullet\rangle$ désigne ici la norme frontière de $L^{2}\left(R^{k-1}\right)$.

Béfinition 1.3. On dit que $P\left(x^{\prime}, t\right)$ satisfait à la condition uniforme de Lopatinski, si les conditions suivantes sont vérifiées 
(i) $p=l \quad$ et

(ii) $\mathbb{P}\left(x^{\prime}, t\right)\left(N^{-} x^{\prime}, 0, t ; \lambda, i \eta\right)$ est non singulier pour tout $\left(x^{\prime}, t\right) \in R^{k-1} \times[0, T], \eta \in R^{k-1}$ et $\lambda$ complexe, $\operatorname{Re} \lambda \geqq 0$.

Théorème 1.2. Supposons que la solution du problème mixte (1.1) avec $g=0$ soit vérifiée (1.3). Alors $P\left(x^{\prime}, t\right)$ satisfait à la condition uniforme de Lopatinski.

Remarque. L'évaluation (1.3) a été dérivée par O.K. Kreiss [3] sous l'hyperbolicité stricte de $L$ et la condition uniforme de Lopatinski. De plus, dans le cas des coefficients constants, Kreiss a déduit que la condition uniforme de Lopatinski est nécessaire.

Le principe de la démonstration du Théorème 1.1. et du Théorème 1.2 . est le même que la méthode de [5], où l'on traite le problème de Cauchy bien posé dans l'espace $L^{2}$.

Daus le cas $m=2$ dans les systèmes (1.1) à coefficients analytiques, on peut obtenir la condition de Lopatinski comme la condition nécessaire, pour que les systèmes (1.1) soient bien posés dans l'espace $\mathscr{E}$, à savoir au sens de la définition suivante.

Définition 1.4. On dit que le problème mixte (1.1) est bien posé dans l'espace $\mathscr{E}$ au voisinage de l'origine, si pour tout voisinage $U$ de l'origine on a un voisinage $\Omega \subset U$ telle que pour tout $f \in C^{\infty}\left(\overline{R_{+}^{k}} \times[0, T]\right)$, $g \in C^{\infty}\left(R_{+}^{k}\right)$ et $h \in C^{\infty}\left(R^{k-1} \times[0, T]\right)$ satisfaisant à la condition de la compatibilité, il existe une solution unique $u \in C^{1}(\bar{\Omega})$ vérifiant

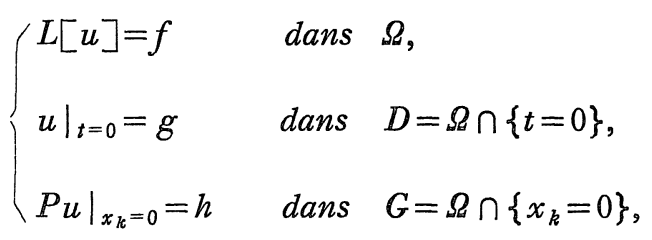

et

$$
|u|_{0, \Omega} \leqq \text { const. }\left\{|g|_{s, D}+|f|_{s, \Omega}+|h|_{s, G}\right\} \text {, }
$$

où $s$ étant un entier posiff, et $|\cdot|_{p, \Omega}, p=0,1,2, \ldots$ désigne la norme naturelle de $C^{p}(\bar{\Omega})$.

Dans le paragraphe 5 , nous allons considérer les systémes (1.1) dont $A_{j}(x, t), j=1,2, \ldots, k$, et $B$ sont des matrices d'ordre 2 et les éléments 
des $A_{j}(x, t), j=1,2, \ldots, k, B$ et $P$ sont des fonctions analytiques. De plus $A_{k}$ et $P$ ont la forme suivante,

$$
\begin{aligned}
& A_{k}=\left(\begin{array}{cc}
a_{1} & 0 \\
0 & a_{2}
\end{array}\right), \quad a_{1}<0, a_{2}>0, \text { et } \\
& P=(a, b), \quad a(0,0) \neq 0,
\end{aligned}
$$

respectivement.

On a alors

Théorème 1.3. Supposons que L et $P$ satisfassent à toutes les conditions énumérées ci-dessus. Si le problème mixte (1.1) est bien posé au sens de la Définition 1.3., alors $P$ satisfait à la condition de Lopatinski à l'origine.

La méthode de la démonstration du Théorème 1.3. doit à P.D. Lax [6].

\section{§2. Préliminaines}

Avant d'entrer dans la démonstration du Théorème 1.1 et 1.2 , nous définissons une suite $\left\{\alpha_{n, \mu}\left(x^{\prime}, t\right)\right\}$ des fonctions, qui jouera un rôle important dans notre raisonnement qui suit. Soit $\alpha\left(x^{\prime}, t\right) \in C_{0}^{\infty}$ tel que supp $[\alpha] \subset R^{k-1} \times(0, T)$ qui vérifie

$$
\int_{0}^{T} \int_{R^{k-1}}\left|\alpha\left(x^{\prime}, t\right)\right|^{2} d x^{\prime} d t=1
$$

Posons pour $n$ et $\mu$ des nombres positives,

$$
\alpha_{n, \mu}\left(x^{\prime}, t\right)=n^{\frac{p}{4}} \alpha\left(n^{\frac{1}{2}} x^{\prime}, n^{\frac{1}{2}} t\right) \exp \left(i x^{\prime} \circ \eta^{0} n+\left(\mu+i \sigma_{0} n\right) t\right),
$$

où $\eta^{0}=\left(\eta_{1}^{0}, \ldots, \eta_{k-1}^{0}\right) \in R^{k-1}, \sigma_{0}$ réel et $x^{\prime} \circ \xi^{0}$ signifie $x_{1} \xi_{1}^{0}+\cdots+x_{k-1} \xi_{k-1}^{0}$.

On va introduire la norme sous la forme suivante,

$$
[u]_{\mu, s}^{2}=\sum_{i+j+|\nu|=s} \int_{0}^{T}\left\|e^{-\mu t} \mu^{i}\left(\frac{\partial}{\partial t}\right)^{i}\left(\frac{\partial}{\partial x}\right)^{\nu} u(t)\right\|^{2} d t
$$

où $s=0,1,2, \ldots, u(t) \in C_{0}^{\infty}\left(\overline{R_{+}^{k}} \times[0, T]\right)$ et $\|\cdot\|$ désigne la norme de $L^{2}\left(R_{+}^{k}\right)$. De plus on définit la norme frontière telle que pour $v \in C_{0}^{\infty}\left(R^{k-1} \times(0, T)\right)$, 


$$
<v>_{\mu, s}^{2}=\int_{R^{k}}\left|\Lambda_{\mu}^{s} v\right|^{2} d x^{\prime} d t
$$

où $s$ étant réel et l'opérateur $\mathbb{A}_{\mu}^{s}$ désigne par

$$
\left(\Lambda_{\mu}^{s} v\right)\left(x^{\prime}, t\right)=\int_{R^{k}} e^{\lambda t+i x^{\prime} \cdot \eta}(\lambda+|\eta|)^{s} \hat{v}(\eta, \lambda) d \sigma d \eta
$$

ici $\lambda=\mu+i \sigma$ et $\hat{v}(\eta, \lambda)$ exprime l'image de Fourier-Laplace par raport à $x^{\prime}$ et $t$, à savoir que

$$
\hat{v}(\eta, \lambda)=\int_{R^{k}} e^{-i x^{\prime} \cdot \eta-\lambda t} v\left(x^{\prime}, t\right) d x^{\prime} d t .
$$

Notons que, car $s$ est un entier non négatif, la norme $\langle v\rangle_{\mu, s}^{2}$ définie par (2.2) équivant à

$$
\sum_{i+j+|\nu|=s} \int_{0}^{T} \int_{R^{k-1}}\left|e^{-\mu t} \mu^{i}\left(\frac{\partial}{\partial t}\right)^{j}\left(\frac{\partial}{\partial x^{\prime}}\right)^{\nu} v\left(x^{\prime}, t\right)\right|^{2} d x^{\prime} d t .
$$

On définit les opérateurs $F_{0}^{(n, \mu)}$ et $F_{j}^{(n)}, j=1,2, \ldots, k-1$, par

$$
\left\{\begin{array}{l}
F_{0}^{(n, \mu)} u=\left(\frac{\partial}{\partial t}-\left(\mu+i \sigma_{0} n\right)\right) u, \text { et } \\
F_{j}^{(n)} u=\left(\frac{\partial}{\partial x_{j}}-i n \eta_{j}^{0}\right) u, j=1, \ldots, k-1,
\end{array}\right.
$$

où $n$ et $\mu$ sont des nombres positifs, $\eta^{0}=\left(\eta_{1}^{0}, \ldots, \eta_{k-1}^{0}\right) \in \mathbb{R}^{k-1}$ et $\sigma_{0}$ réel. Dans ce qui suit, pour simplifier, nous écrirons $F_{0}$ et $F_{j}(j=1, \ldots, k-1)$ au lieu de $F_{0}^{(n, \mu)}$ et de $F_{j}^{(n)}$ respectivement.

Ceci préparé, on a facilement le

Lemame 2.1. Soit $\alpha_{n, \mu}\left(x^{\prime}, t\right)$ défini par (2.1). Alors on a pour $n$ et $\mu$ des nombres positifs arbitraires, $\mu \leqq n$ et pour $s$ le nombre non négatif,

(i) $\left\langle\alpha_{n, \mu}\right\rangle_{\mu, 0}=1$,

(ii) $\left\langle\alpha_{n, \mu}\right\rangle_{\mu, s} \leqq$ const. $n^{s}$,

(iii) $\sum_{j=0}^{k-1}\left\langle x_{j} \alpha_{n, \mu}\right\rangle_{\mu, s} \leqq$ const. $n^{s-\frac{1}{2}}$

où on a écrit $t=x_{0}$,

$$
\text { (iv) } \begin{aligned}
<F_{0}^{(n, \mu)} \alpha_{n, \mu}>_{\mu, s} \leqq \text { const. } n^{s+\frac{1}{2},} \\
<F_{j}^{(n)} \quad \alpha_{n, \mu}>_{\mu, s} \leqq \text { const. } n^{s+\frac{1}{2},}
\end{aligned}
$$


où toutes les constantes sont indépendantes de $\mu$ et de $n$.

Preuve. (i) est évident d'après la définition de $\alpha_{n, \mu}$. (ii). On peut écrire,

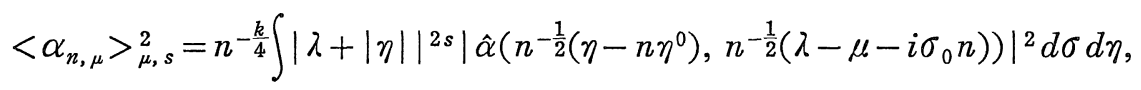

où $\hat{\alpha}(\eta, \lambda)$ est l'image de Fourier-Laplace. Cela entraîne l'assertion (ii). (iii). Puisqu'on a

$$
\begin{aligned}
& <x_{j} \alpha_{n, \mu}>_{\mu, s}^{2}=n-\frac{k}{4} \int|\lambda+| \eta|| 2 s \mid n^{-\frac{1}{2}} \hat{\alpha}_{\eta,}\left(n^{-\frac{1}{2}}\left(\eta-n \eta^{0}\right),\right. \\
& \left.n-\frac{1}{2}\left(\lambda-\mu-i \sigma_{0} n\right)\right)\left.\right|^{2} d \sigma d \eta
\end{aligned}
$$

on peut obtenir (iii). Enfin, notons qu'on a

$$
F_{j} \alpha_{n, \mu}=n^{\frac{1}{2}+\frac{k}{4}} \exp \left(i x^{\prime} \cdot \eta^{0} n+\left(\mu+i \sigma_{0} n\right) t\right) \alpha_{x_{j}}\left(n^{\frac{1}{2}} x^{\prime}, n^{\frac{1}{2}} t\right),
$$

on obtient (iv).

Dans la suite, on pose

$$
\beta_{n}\left(x^{\prime}, t\right)=n^{\frac{k}{4}} \alpha\left(n^{\frac{1}{2}} x^{\prime}, n^{\frac{1}{2}} t\right) e^{n t}
$$

Corollaire 2.1。 La fonction $\beta_{n}\left(x^{\prime}, t\right)$ vérifie,

(i) $\left\langle\beta_{n}\right\rangle_{n, 0}=1$

(ii) $\sum_{j=1}^{k-1}\left\langle\frac{\partial}{\partial x_{j}} \beta_{n}\right\rangle_{n, \frac{1}{2}}+\sum_{j=0}^{k-1}\left\langle x_{j} \beta_{n}\right\rangle_{n, \frac{3}{2}} \leqq$ const. $n$

(iii) $\left\langle\left(\frac{\partial}{\partial t}-n\right) \beta_{n}\right\rangle_{n, \frac{1}{2}} \leqq$ const. $n$,

où toutes les constantes sont indépendantes de $n$.

\section{§3. La Comdition de Lopatingski}

Dans ce paragraphe, nous allons établir le Théorème 1.2. Pour cela, nous utiliserons l'inégalité de l'énergie dérivé de l'inégalité (1.2).

Considérons le problème mixte (1.1) avec $g=0$, c'est-à-dire, 


$$
\left\{\begin{array}{l}
L[u]=f, \\
\left.u\right|_{t=0}=0, \\
\left.P u\right|_{x_{k}=0}=g .
\end{array}\right.
$$

Alors on a

Proposition 3.H. Supposons que le problème mixte $(L, P)$ soit bien posé dans l'espace $L^{2}$. Alors il exsite une solution du problème (3.1) pour tout $f \in C_{0}^{\infty}\left(R_{+}^{k} \times(0, T)\right)$ et $h \in C_{0}^{\infty}\left(R^{k-1} \times(0, T)\right)$, supp $[h]$ assez petit, telle qu'on ait, pour $\mu>0$,

$$
\mu[u]_{\mu, 0} \leqq \text { const. }\left\{[f]_{\mu, 0}+\langle h\rangle_{\mu, \frac{1}{2}}\right\},
$$

plus généralement, on a pour s un entier positif

$$
\mu[u]_{\mu, s} \leqq \text { const. }\left\{[f]_{\mu, s}+\langle h\rangle_{\mu, s+\frac{1}{2}}\right\},
$$

où const. est indépendant de $\mu$.

Preuve. Puisque le rang de $P$ est constant et supp $[h]$ est assez petit, on peut construire une fonction $v \in H^{1}\left(R_{+}^{k} \times(0, T)\right)$ telle que

$$
\begin{aligned}
& \left.P v\right|_{x_{k=0}}=h, \text { et } \\
& {[v]_{\mu, 1} \leqq \text { const. }\left\langle h>_{\mu, \frac{1}{2}} .\right.}
\end{aligned}
$$

De l'hypothèse il découle qu'il existe une solution $w$ pour l'équation suivante

$$
\begin{aligned}
& L[w]=f-L[v], \\
& \left.w\right|_{t=0}=0, \\
& \left.P w\right|_{x_{k}=0}=0,
\end{aligned}
$$

et de plus on a

$$
\|w(t)\| \leqq \text { const. } \int_{0}^{t}(\|f(s)\|+\|L[v](s)\|) d s .
$$

En le multipliant par $e^{-\mu t}$ et intégrant en $t$, on obtient, d'après l'inégalité de Schwarz, 


$$
\mu[w]_{\mu, 0} \leqq \text { const. }\left\{[f]_{\mu, 0}+[v]_{\mu, 1}\right\}
$$

qui et (3.4) entraînent (3.2). Généralement, on remarque que $\Lambda_{\mu}^{s} u$ saitsfait à

$$
\begin{aligned}
& L\left[\Lambda_{\mu}^{s} u\right]=A_{k} \Lambda_{\mu}^{s} A_{k}^{-1} f+A_{k}\left[M, \Lambda_{\mu}^{s}\right] u, \\
& \left.\Lambda_{\mu}^{s} u\right|_{t=0}=0, \\
& \left.P \Lambda_{\mu}^{s} u\right|_{x_{k}=0}=\left.\left[\Lambda_{\mu}^{s}, P\right] u\right|_{x_{k}=0}+\Lambda_{\mu}^{s} h,
\end{aligned}
$$

où $M=A_{k}^{-1}\left(\frac{\partial}{\partial t}-\sum A_{j} \frac{\partial}{\partial x_{j}}\right) . \quad\left[M, \Lambda_{\mu}^{s}\right]$ et $\left[\Lambda_{\mu}^{s}, P\right]$ sont des opérateurs pseudo-différentiels d'ordre $s$ et $s-1$ respectivement. Donc, en appliquant (3.2) à $\Lambda_{\mu}^{s} u$, on a (3.3).

Dans la suite, on considère le problème mixte suivant,

$$
\left\{\begin{array}{l}
L[u]=0, \\
\left.u\right|_{t=0}=0, \\
\left.P u\right|_{x_{k}=0}=h,
\end{array}\right.
$$

où $h \in C_{0}^{\infty}\left(R^{k-1} \times(0, T)\right)$ et supp $[h]$ assez petit.

On a alors, en vertu de la Proposition 3.1.

Proposition 3.2. Supposons que $(L, P)$ soit bien posé dans l'espace $L^{2}$. Alors la solution $u$ du problème mixte (3.5) satisfait à

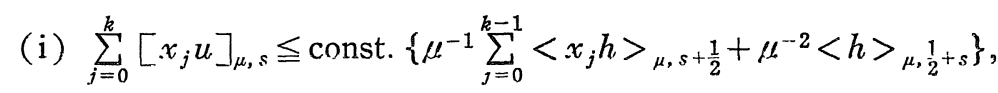
ò̀ on a écrit $t=x_{0}$,

(ii) $\sum_{j=0}^{k-1}\left[F_{j} u\right]_{\mu, 0} \leqq$ const. $\left\{\ell^{-1} \sum_{j=0}^{k-1}\left\langle F_{j} h>_{\mu, \frac{1}{2}}+\ell^{-2}<h>_{\mu, \frac{3}{2}}\right\}\right.$, où les $F_{j}$ se définissent par (2.3), et toutes les constantes sont indépendantes de $n$ et $\mu$.

Preuve. (i), en multipliant (3.5) par $x_{j},(j=0,1, \ldots, k)$, on a

$$
L\left[x_{j} u\right]=Q u
$$




$$
\begin{aligned}
& \left.x_{j} u\right|_{t=0}=0, \\
& P\left[x_{j} u\right]_{x_{k}=0}=x_{j} h,
\end{aligned}
$$

où $Q$ est un opérateur borné. En vertu de la Proposition 3.1, on a

$$
\begin{aligned}
{\left[x_{j} u\right]_{\mu, s} } & \leqq \text { const. } \mu^{-1}\left\{\left\langle x_{j} h\right\rangle_{\mu, \frac{1}{2}+s}+[u]_{\mu, s}\right\}, \\
& \left.\leqq \text { const. }\left\{\mu^{-1}\left\langle x_{j} h\right\rangle_{\mu, \frac{1}{2}+s}+\mu^{-2}<h\right\rangle_{\mu, s+\frac{1}{2}}\right\}
\end{aligned}
$$

où on remarque que $x_{j} h$ s'annule pour $j=k$. (ii), faisons opérer $F_{j}$ à (3.5), on a

$$
\begin{aligned}
& L\left[F_{j} u\right]=B_{j} u, \\
& \left.F_{j} u\right|_{t=0}=0, \\
& \left.P\left[F_{j} u\right]\right|_{x_{k}=0}=F_{j} h+Q u,
\end{aligned}
$$

où $B_{j}(j=0,1, \ldots, k-1)$ sont des opérateurs différentiels du premier ordre et un opérateur $Q$ borné. Donc, compte tenu de (3.2), on a

$$
\begin{aligned}
{\left[F_{j} u\right]_{\mu, 0} } & \leqq \text { const. } \mu^{-1}\left\{\left\langle F_{j} h\right\rangle_{\mu, \frac{1}{2}}+[u]_{\mu, 1}+\langle u\rangle_{\mu, \frac{1}{2}}\right\} \\
& \leqq \text { const. } \mu^{-1}\left\{\left\langle F_{j} h\right\rangle_{\mu, \frac{1}{2}}+[u]_{\mu, 1}\right\}
\end{aligned}
$$

d'où on obtient (ii), en utilisant (3.3).

Corollaire 3.1. On a

$$
\begin{aligned}
& \text { (i) } \sum_{j=1}^{k-1}\left[\frac{\partial}{\partial x_{j}} u\right]_{\mu, 0} \leqq \text { const. }\left\{\mu^{-1} \sum_{j=1}^{k-1}\left\langle\frac{\partial}{\partial x_{j}} h\right\rangle_{\mu, \frac{1}{2}}+\mu^{-2}\langle h\rangle_{\mu, \frac{3}{2}}\right\}, \\
& \text { (ii) } \left.\left[\left(\frac{\partial}{\partial t}-n\right) u\right]_{\mu, 0} \leqq \text { const. }\left\{\mu^{-1}\left\langle\left(\frac{\partial}{\partial t}-n\right) h\right\rangle_{\mu, \frac{1}{2}}+\mu^{-2}<h\right\rangle_{\mu, \frac{3}{2}}\right\} .
\end{aligned}
$$

Démonstration $d u$ Théorème 1.1. D'abord on va montre que $l=p$. Supposons que $l>p$. On décompose

$$
P(0,0)=\left(\begin{array}{ll}
P_{11}, & P_{12} \\
P_{21}, & P_{22}
\end{array}\right),
$$

où $P_{11}$ est une matrice d'ordre $p$. On prouve que $P_{11}$ est non singulier. 
Si $P_{11}$ est singulier, on a la matrice $T$ non singulière d'ordre $p$ telle que

$$
T P_{11} T^{-1}=\left(\begin{array}{ccc} 
& R_{0} \\
& & \\
0 & \ldots & 0
\end{array}\right),
$$

où $R_{0}$ est une matrice à $p-1$ lignes et $p$ colonnes. On pose

$$
v=\left(\begin{array}{llll}
T & & & 0 \\
& & & \\
& 1 & & \\
& & \ddots & \\
0 & & & 1
\end{array}\right) u \equiv S u .
$$

Alors (3.5) s"ecrit

$$
\begin{aligned}
& \frac{\partial}{\partial x_{k}} v=n S A^{-1}(0,0) S{ }^{1} v+f, \\
& \left.P(0,0) S^{-1} v\right|_{x_{k}=0}+\varphi=h,
\end{aligned}
$$

où $\quad f=S\left(A^{-1} \frac{\partial}{\partial t}-n A^{-1}(0,0)\right) u-S A^{-1} \Sigma A_{j} \frac{\partial}{\partial x_{j}} u$ et

$$
\varphi=\left.(P-P(0,0)) u\right|_{x_{k}=0} .
$$

On remarque que

$$
\begin{aligned}
& {[f]_{n, 0} \leqq \text { const. } }\left\{\sum_{j=0}^{k}\left[x_{j} u\right]_{n, 1}+\left[\left(\frac{\partial}{\partial t}-n\right) u\right]_{n, 0}\right. \\
&\left.+\sum_{j=1}^{k-1}\left[\frac{\partial}{\partial x_{j}} u\right]_{n, 0}\right\} \text { et } \\
&<\varphi>_{n, 0} \leqq \text { const. } \sum_{j=0}^{k-1}\left\langle x_{j} u>_{n, 0}\right. \\
& \leqq \text { const. }\left\{\sum_{j=0}^{k-1} n^{-1 / 2}\left[x_{j} u\right]_{n, 1}+n^{1 / 2}\left[x_{j} u\right]_{n, 0}\right\} .
\end{aligned}
$$

On écrit $v={ }^{t}\left(v_{1}, \ldots, v_{p}, v_{p+1}, \ldots, v_{m}\right)={ }^{t}\left(v^{(1)}, v^{(2)}\right)$. Alors on a

$$
v^{(2)}=n A_{+}^{-1} v^{(2)}+f^{(2)} \text {. }
$$

Puisque toutes les valeurs propres de $A_{+}$sont positives, on a

$$
<v^{(2)}>_{n, 0}^{2}+n\left[v^{(2)}\right]_{n, 0}^{2} \leqq \text { const. } \frac{1}{n}\left[f^{(2)}\right]_{n, 0}^{2} \text {. }
$$


Maintenant on choisit $h$ tel que $(T, 0) h={ }^{t}\left(0, \ldots, 0, h_{p}, 0 \ldots 0\right)$. Alors compte tenu de (3.6), de (3.7), de (3.8), de (3.9) et de (3.10), on a

$$
\begin{aligned}
\left.<h_{p}\right\rangle_{n, 0} \leqq \text { const. }\left\langle v^{(2)}>_{n, 0}+\left\langle\varphi>_{n, 0},\right.\right. \\
\leqq \text { const. }\left\{n^{-1 / 2}\left(\sum_{j=0}^{k}\left[x_{j} u\right]_{n, 1}+\sum_{j=1}^{k-1}\left[\frac{\partial}{\partial x_{j}} u\right]_{n, 0}+\left[\left(\frac{\partial}{\partial t}-n\right) u\right]_{n, 0}\right)\right. \\
\left.\sum_{j=0}^{k-1} n^{1 / 2}\left[x_{j} u\right]_{n, 0}\right\},
\end{aligned}
$$

d'où l'on a en utilisant le Proposition 3.2 et le Corollaire 3.1 avec $\mu=n$,

$$
\begin{aligned}
\left\langle h_{p}\right\rangle_{n, 0} \leqq \text { const. } & \left\{n ^ { - 3 / 2 } \left(\sum_{j=1}^{k-1}\left\langle x_{j} h_{p}\right\rangle_{n, 3 / 2}+\sum_{j=1}^{k-1}\left\langle\frac{\partial}{\partial x_{j}} h_{p}\right\rangle_{n, \frac{1}{2}}\right.\right. \\
& \left.\left.+\left\langle\left(\frac{\partial}{\partial t}-n\right) h_{p}\right\rangle_{n, \frac{1}{2}}\right)+n^{-1 / 2}<h_{p}\right\rangle_{n, \frac{1}{2}} \\
& +n^{-5 / 2}\left\langle h_{p}\right\rangle_{n, \frac{3}{2}}+n^{-1 / 2} \sum_{j=1}^{k-1}\left\langle x_{j} h_{n, \frac{1}{2}}\right\} .
\end{aligned}
$$

On ici choisit $h_{p}=\beta_{n}\left(x^{\prime}, t\right)$ défini par (2.4) comme $h_{p}$. Alors, le terme à droite de (3.11) se majore par const. $n^{-1 / 2}$ en vertu du Lemme 2.1 et du Corollaire 2.1. D'autre part, le terme à gauche de (3.11) prend 1 d'après (i) du Corollaire 2.1. Cela implique contradiction pour $n$ assez grand. Ce prouve ainsi que $P_{11}$ est nonsingulier.

Maintenant nous revenons de nouveau au problème (3.7). On décompose $S^{-1} v=u={ }^{t}\left(u^{(1)}, u^{(2)}\right)$. Alors (3.7) se récrit

$$
\frac{\partial}{\partial x_{k}} u=n\left(\begin{array}{cc}
A_{-}^{-1} & 0 \\
0 & A_{+}^{-1}
\end{array}\right)\left(\begin{array}{l}
u^{(1)} \\
u^{(2)}
\end{array}\right)+f,
$$

$$
\left(\begin{array}{ll}
P_{11} & P_{12} \\
P_{21} & P_{22}
\end{array}\right)\left(\begin{array}{l}
u^{(1)} \\
u^{(2)}
\end{array}\right)_{x_{k}=0}+\varphi=h .
$$

Puisque $P_{11}$ est non singulier, d'après (3.12) on a

$$
h^{(2)}=\varphi^{(2)}+\left.P_{22} u^{(2)}\right|_{x_{k}=0}+P_{21} P_{11}^{-1}\left(h^{(1)}-\varphi^{(1)}-\left.P_{12} u^{(2)}\right|_{x_{k}=0}\right),
$$

d'où, on peut évaluer, en choisissant $h=\left(0, \ldots, 0, \beta_{n}\right)$, 


$$
\left\langle\beta_{n}\right\rangle_{n, 0} \leqq \text { const. }\left\{\left\langle u^{(2)}\right\rangle_{n, 0}+\langle\varphi\rangle_{n, 0}\right\} \text {. }
$$

Notons que $u^{(2)}$ satisfait à (3.10), de la même manière ci-dessus on a l'inégalité (3.11). Cela implique une contradiction. Donc, on a montré que $l \leqq p$.

Ensuite, supposons que $l<p$. On décompose

$$
P(0,0)=\left(P_{1}, P_{2}\right)
$$

où $P_{1}$ est une matrice à $l$ lignes et $p$ colonnes. Puisque $l<p$, il existe un vecteur $w={ }^{t}\left(w_{1}, \ldots, w_{p}\right) \neq 0$ tel que

$$
P_{1} w=0
$$

On note que $A_{k}(0,0)$ a la forme suivante

$$
A_{k}(0,0)=\left(\begin{array}{ccccc}
a_{1} & & & & \\
& \ddots & & & \\
& & a_{p} & & \\
& & & & \\
& & a_{p+1} & \\
& & & \ddots & \\
0 & & & & a_{m}
\end{array}\right),
$$

où $a_{j},(j=1, \ldots, p)$, sont réels négatifs et $a_{j},(j=p+1 \ldots, m)$ réels positifs. On pose

$$
u(x, t)=\sum_{i=1}^{P} c_{i} n^{k / 4} \exp \left(a_{i}^{-1} n x+n t\right) \alpha\left(n^{1 / 2} x^{\prime}, n^{1 / 2} t\right) e_{i}
$$

où $e_{j}={ }^{t}\left(0, \ldots, 0,1^{j}, 0 \ldots 0\right), j=1,2, \ldots, p$ sont des vecteurs dan $R^{m}$. Alors on a

$$
[u]_{n, 0}^{2}=\sum_{i=1}^{p}\left|c_{i} a_{i}\right|^{2} \neq 0
$$

En appliquant $\left(\frac{\partial}{\partial x_{k}}-M\right)$ à la fonction $u(x, t)$, on a

$$
\left(\frac{\partial}{\partial x_{k}}-M\right) u=\left(A^{-1}(0,0)-A^{-1}(x, t)\right) \frac{\partial}{\partial t} u+A_{k}^{-1} \sum_{j=1}^{k-1} A_{j} \frac{\partial}{\partial x_{j}} u \equiv f .
$$

De plus (3.13), on obtient

$$
\left.P u\right|_{x_{k}=0}=\left.(P(x, t)-P(0,0)) u\right|_{x_{k}=0} \equiv h .
$$


Donc, de la définition de $u(x, t)$, on peut évaluer,

$$
[f]_{n, 0} \leqq \text { const. } n^{1 / 2} \text {, et }
$$

$$
<h>_{n, 1 / 2} \leqq \text { const. } n^{1 / 2} \text {. }
$$

D'autre part, puisque $(L, P)$ est bien posé dans l'espace $L^{2}$, on a, compte tenu de (3.15),

$$
\begin{aligned}
{[u]_{n, 0} } & \leqq \text { const. } \frac{1}{n}\left\{[f]_{n, 0}+<h>_{n, 1 / 2}\right\} \\
& \leqq \text { const. } n^{-1 / 2}
\end{aligned}
$$

qui n'est pas compatible avec (3.14) pour $n$ assez grand. Cela prouve que $p=l$.

Dans la suite, on va prouver que la propriété (ii) de la Définition 1.1, c'est-à-dire,

$$
\text { dét } P N^{-}\left(x^{\prime}, t ; \lambda, i \eta\right) \neq 0
$$

pour tout $\lambda$ complexe, $\operatorname{Re} \lambda>0, \eta \in R^{k-1}$ et $\left(x^{\prime}, t\right) \in R^{k-1} \times[0, T]$.

Supposons que $P N^{-}$est singulier pour $\lambda_{0}, \operatorname{Re} \lambda_{0}>0, \eta^{0} \in R^{k-1}$ et $\left(x_{0}, t_{0}\right)$. Pour simplifier, on prend $\left(x_{0}, t_{0}\right)=(0,0)$. On considère le problème mixte (3.5). On pose $N^{-1}\left(0,0 ; \lambda_{0}, i \eta^{0}\right) u=v={ }^{t}\left(v_{1}, \ldots, v_{l}, v_{l+1}, \ldots, v_{m}\right)={ }^{t}\left(v^{(1)}, v^{(2)}\right)$. Alors (3.5) s'ecrit,

$$
\frac{\partial}{\partial x_{k}} v=n\left(\begin{array}{cc}
M^{-}\left(0,0 ; \lambda_{0}, i \eta^{0}\right) & * \\
0 & M^{+}\left(0,0 ; \lambda_{0}, i \eta^{0}\right)
\end{array}\right) v+f,
$$

$$
\left.P(0,0) N\left(0,0 ; \lambda_{0}, i \eta^{0}\right) v\right|_{x_{k}=0}+\varphi=h
$$

où $f$ et $\varphi$ expriment,

$$
\begin{aligned}
& f=N^{-1}\left(M\left(x, t ; \frac{\partial}{\partial t}, \frac{\partial}{\partial x^{\prime}}\right)-n M\left(0,0 ; \lambda_{0}, i \eta^{0}\right)\right) u, \text { et } \\
& \varphi=\left.\left(P\left(x^{\prime}, t\right)-P(0,0)\right) u\right|_{x_{k}=0},
\end{aligned}
$$

respectivement. Nous notons que nous avons

$$
[f]_{\mu, 0} \leqq \text { const. }\left\{\sum_{j=0}^{k}\left[x_{j} u\right]_{\mu, 1}+\sum_{j=0}^{k-1}\left[F_{j} u\right]_{\mu, 0}\right\} \text { et }
$$


(3.18) $<\varphi>_{\mu, 0} \leqq$ const. $\left\{\sum_{j=0}^{k-1}<x_{j} u>_{\mu, 0}\right\}$,

$$
\leqq \text { const. }\left\{n^{-1 / 2} \sum_{j=0}^{k-1}\left[x_{j} u\right]_{\mu, 1}+n^{1 / 2} \sum_{j=0}^{k-1}\left[x_{j} u\right]_{\mu, 0}+n^{-1 / 2}[u]_{\mu, 0}\right\}
$$

La dernière inégalité peut se prouver de la manière suivante,

$$
\begin{aligned}
\left\langle x_{j} u>{ }_{\mu, 0}^{2}\right. & =-2 \operatorname{Re} \int_{0}^{T}\left(e^{-\mu t}\left(M x_{j} u+B u\right), e^{-\mu t} x_{j} u\right) d t, \\
& \leqq \text { const. }\left\{\frac{1}{n}[M u]_{\mu, 0}^{2}+n\left[x_{j} u\right]_{\mu, 0}^{2}+\frac{1}{n}[u]_{\mu, 0}^{2}\right\} .
\end{aligned}
$$

Soit $w$ le vecteur-nul à ganche de $P N^{-}\left(0,0 ; \lambda_{0}, i \eta^{0}\right)$, c'est-à-dire

$$
\text { w. } P N^{-}\left(0,0 ; \lambda_{0}, i \eta^{0}\right)=0
$$

Maintenant on choisit $h$ tel que $w . h=\alpha_{n, \mu}$, qui se définit par (2.1). En multipliquant par $w$ le second terme de (3.16), d'après (3.19) on a

$$
\alpha_{n, \mu}=\left.w \cdot P N^{+}\left(0,0 ; \lambda_{0}, i \eta^{0}\right) v^{(2)}\right|_{x_{k}=0}+\varphi^{(2)},
$$

d'où l'on peut évaluer

$$
\left.\left.<\alpha_{n, \mu}\right\rangle_{\mu, 0} \leqq \text { const. }\left\{\left\langle v^{(2)}\right\rangle_{\mu, 0}+<\varphi^{(2)}\right\rangle_{\mu, 0}\right\} .
$$

Puisque $M^{+}\left(0,0 ; \lambda_{0}, i \eta^{0}\right)$ a seulement les valeurs propres à partie réelle positive, compte tenu du premier terme de (3.16), on obtient

$$
<v^{(2)}>_{\mu, 0}^{2}+n\left[v^{(2)}\right]_{\mu, 0}^{2} \leqq \text { const. } n^{-1}\left[f^{(2)}\right]_{\mu, 0}^{2}
$$

qui et (3.20) entraînent

$$
<\alpha_{n, \mu}>_{\mu, 0} \leqq \text { const. }\left\{n^{-1 / 2}[f]_{\mu, 0}+<\varphi^{(2)}>_{\mu, 0}\right\}
$$

On pose $\mu=\operatorname{Re} \lambda_{0} \cdot n$. Alors, d'après (3.17), (3.18) et Proposition 3.2, on dérive de (3.21)

$$
\begin{aligned}
<\alpha_{n, \mu}>_{\mu, 0} \leqq \text { const. } & \left\{n ^ { \frac { 3 } { 2 } } \sum _ { j = 0 } ^ { k - 1 } \left(<x_{j} \alpha_{n, \mu}>_{\mu, \frac{3}{2}}+<F_{j} \alpha_{n, \mu}>_{\mu, \frac{1}{2}}\right.\right. \\
+ & \left.\left.n<x_{j} \alpha_{n, \mu}>_{\mu, \frac{1}{2}}\right)+n^{-5 / 2}<\alpha_{n, \mu}>_{\mu, \frac{3}{2}}\right\} .
\end{aligned}
$$


Compte tenu du Lemme 2.1 avec $\mu=\operatorname{Re} \lambda_{0} n$, le terme à gauche de (3.22) est égale à 1 et terme à droite se majore par const. $n^{-\frac{1}{2}}$. Cela implique une contradition pour $n$ assez grand. Ce prouve le Théorème 1.1.

\section{§4. La Condition Uniforme de Lopatinski}

Dans ce paragraphe, nous allons établir le Théorème 1.2, qui, se prouve de la même façon que le Théorème 1.1.

Nous supposons ici que $(L, P)$ soit bien posé dans 1'espace $L^{2}$ et de plus la solution du problème mixte suivant,

$$
\left\{\begin{array}{l}
L[u]=f, \\
\left.u\right|_{t=0}=0, \\
\left.P u\right|_{x_{k}=0}=h,
\end{array}\right.
$$

vérifie (1.2) pour tout $\mu>0$, c'est-à-dire,

$$
\mu[u]_{\mu, 0}^{2}+<u>_{\mu, 0}^{2} \leqq \text { const. }\left\{\mu^{-1}[f]_{\mu, 0}^{2}+<h>_{\mu, 0}^{2}\right\}
$$

pour tout $f \in C_{0}^{\infty}\left(R_{+}^{k} \times(0, T)\right)$ et tout $h \in C_{0}^{\infty}\left(R^{k-1} \times(0, T)\right)$.

Il découle facilement de (4.2) que l'on a

Proposition 4.1. Supposons que (4.2). Alors il existe une constante $\mu_{s}$ pour $s$ entier non négatif telle que pour $f \in C_{0}^{\infty}\left(R_{+}^{k} \times(0, T)\right)$ et $h \in C_{0}^{\infty}$ $\left(R^{k-1} \times(0, T)\right)$,

$$
\mu[u]_{\mu, s}^{2}+<u>_{\mu, s}^{2} \leqq \text { const. }\left\{\mu^{-1}[f]_{\mu, s}^{2}+<h>_{\mu, s}^{2}\right\}
$$

pour $\mu \geqq \mu_{s}$.

Maintenant, nous considérons le problème mixte (4.1) avec $f=0$, à savoir,

$$
\left\{\begin{array}{l}
L[u]=0 \\
\left.u\right|_{t=0}=0 \\
\left.P u\right|_{x_{k}=0}=h
\end{array}\right.
$$


Alors on a

Proposition 4.2. Supposons que $(L, P)$ vérifie l'évalution (4.2). Alors la solution de (4.4) satisfait aux suivants

(i ) $\mu \sum_{j=0}^{k}\left[x_{j} u\right]_{\mu, s}^{2}+\sum_{j=0}^{k-1}<x_{j} u>_{\mu, s}^{2} \leqq$ const. $\left\{\sum_{j=0}^{k-1}<x_{j} h>_{\mu, s}^{2}+\frac{1}{\mu}<h>_{\mu, s}^{2}\right\}$ pour tout $\mu \geqq \mu_{s}, s$ étant un entier non négatif, et on a écrit $x_{0}=t$,

(ii) $\sum_{j=0}^{k-1} \mu\left[F_{j} u\right]_{\mu, 0}^{2}+<F_{j} u>_{\mu, 0}^{2} \leqq$ const. $\left\{\sum_{j=0}^{k-1}<F_{j} h>_{\mu, 0}^{2}+\mu^{-1}<h>_{\mu, 0}^{2}\right\}$, pour $n \geqq \mu$, où l'on définit $F_{j}$ par (2.3).

Cette proposition se prouve par la même manière que le Proposition 3.2. Démonstration du Théorème 1.2. Nous allons prouver ce théorème par contradiction. Supposons que dét $\left(P N^{-}\right)$soit nul pour $\left(x_{0}^{\prime}, t_{0}\right)$, et $\left(\lambda_{0}, \eta^{0}\right), \operatorname{Re} \lambda_{0} \geqq 0$. Il découle du Théorème 1.1 que la partie réelle de $\lambda_{0}$ est zèro. On écrit $\lambda_{0}=i \sigma_{0}$. Pour simplifier notre resonnement, supposons désormais que $\left(x_{0}^{\prime}, t_{0}\right)=(0,0)$. On pose

$$
R(\varepsilon)=P N^{-}\left(0,0 ; \varepsilon+i \sigma_{0}, i \eta^{0}\right), \quad \varepsilon \geqq 0 .
$$

Puisque les éléments des $N$ se constituent en des fonctions continues, $R(\varepsilon)$ varie continûment en $\varepsilon$. Donc, de l'hypothése il découle que $R(0)$ est singulier. Soit $c=\left(c_{1}, \ldots, c_{l}\right)$ un vecteur-nul à gauche de $R(0)$. On pose $w(\varepsilon)=c R(\varepsilon)$. Alors, par continuité, on a

$$
|w(\varepsilon)| \rightarrow 0 \text {, quand } \varepsilon \rightarrow 0 \text {. }
$$

Maintenent, nous revenons de nouveau au problème mixte (4.4). On pose $v=N^{-1}\left(0,0 ; \mu / n+i \sigma_{0}, i \eta^{0}\right) u=\left(v^{(1)}, v^{(2)}\right)$. Alors, on peut écrire à la maniére suivante

$$
\frac{\partial}{\partial x_{k}} v=n\left(\begin{array}{cc}
M^{-}\left(0,0 ; \mu / n+i \sigma_{0}, i \eta^{0}\right) & * \\
0 & M^{+}\left(0,0 ; \mu / n+i \sigma_{0}, i \eta^{0}\right)
\end{array}\right) v+f,
$$

$$
\left.R(\mu / n) v^{(1)}\right|_{x_{k}=0}+\left.P N^{+} v^{(2)}\right|_{x_{k}=0}+\varphi=h,
$$

où $f$ et $\varphi$ expriment 


$$
\begin{aligned}
& f=N^{-1}\left(0,0 ; \mu / n+i \sigma_{0}, i \eta^{0}\right)\left(M\left(x, t ; \frac{\partial}{\partial t}, \frac{\partial}{\partial x^{\prime}}\right)\right. \\
& -n M\left(0,0 ; \mu / n+i \sigma_{0}, i \eta^{0}\right) u \text { et } \\
& \varphi=\left.\left(P\left(x^{\prime}, t\right)-P(0,0)\right) u\right|_{x_{k}=0}
\end{aligned}
$$

On ici choisit $h$ tel que $c . h=\Sigma c_{i} h_{i}=\alpha_{n, \mu}\left(x^{\prime}, t\right)$ défini par (2.1). En multipliant par $c$ le second membre de (4.6), on a

$$
\alpha_{n, \mu}=\left.w(\mu / n) v^{(1)}\right|_{x_{k}=0}+\left.c \cdot P N^{+}\left(0,0 ; \mu / n+i \sigma_{0}, i \eta^{0}\right) v^{(2)}\right|_{x_{k}=0}+c \cdot \varphi,
$$

d'où l'on peut évaluer

$$
\left.<\alpha_{n, \mu}>_{\mu, 0} \leqq|w(\mu / n)|<v^{(1)}\right\rangle_{\mu, 0}+\text { const. }\left(\left\langle v^{(2)}\right\rangle_{\mu, 0}+<\varphi>_{\mu, 0}\right),
$$

où const. est independant de $\mu$ et de $n$. En utilisant le premier membre de (4.6), nous allons évaluer $v^{(2)}$, qui satisfait à

$$
v^{(2)}=n M^{+}(\mu / n) v^{(2)}+f^{(2)}
$$

où $M^{+}(\mu / n)=M^{+}\left(0,0 ; \mu / n+i \sigma_{0}, i \eta^{0}\right)$. Nous notons qu'il découle de l'hyperbolicité de $L$ que la partie réelle des racines caractéristiques de $M^{+}(\mu / n)$ se minore par const. $\mu / n$. On a donc pour tout $\xi \in R^{1}$,

$$
\left|(i \xi-M(\mu / n))^{-1}\right| \leqq \text { const. }(n / \mu)^{m-l} \times(|\xi|+\mu)^{-1} \text {. }
$$

On represente, du (4.9),

$$
v^{(2)}\left(x^{\prime}, 0, t\right)=\frac{1}{2 \pi} \int_{-\infty}^{\infty}(i \xi-M(\mu / n))^{-1} \hat{f}^{(2)}(\xi) d \xi
$$

où $\hat{f}(\xi)$ exprime l'image de Fourier par rapport à $x_{k}$, à savoir,

$$
\widehat{f}^{(2)}(\xi)=\int_{0}^{\infty} e^{-i x_{k} \xi} f^{(2)}\left(x_{k}\right) d x_{k}
$$

Compte tenu de (4.11) on a, en utilisant (4.10) et l'inéqualité de Schwarz,

$$
\left.<v^{(2)}\right\rangle_{\mu, 0} \leqq \text { const. }(n / \mu)^{m-l} \mu^{-\frac{1}{2}}\left[f^{(2)}\right]_{\mu, 0},
$$

d'où, on a en vertu de (4.9),

$$
\begin{aligned}
<\alpha_{n, \mu}>_{\mu, 0} \leqq \text { const. }\{|w(\mu / n)|<u\rangle_{\mu, 0} \\
\\
\left.+(n / \mu)^{m-l} \mu^{-\frac{1}{2}}[f]_{\mu, 0}+<\varphi>_{\mu, 0}\right\} .
\end{aligned}
$$


Notons que $f$ et $\varphi$ vérifient les inégalités (3.22) et (3.23) respéctivement. Donc, on a finalement d'après (4.12) et Proposition 4.2,

$$
\begin{aligned}
& <\alpha_{n, \mu}>_{\mu, 0} \leqq \text { const. }\left\{|w(\mu / n)|<\alpha_{n, \mu}>_{\mu, 0}+\sum_{j=0}^{k-1}<x_{j} \alpha_{n, \mu}>_{\mu, 0}\right. \\
& +\mu^{-\frac{1}{2}}<\alpha_{n, \mu}>_{\mu, 0}+(n / \mu)^{m \cdots l} \mu^{-\frac{1}{2}}\left(\sum _ { j = 0 } ^ { k - 1 } \left(\left\langle x_{j} \alpha_{n, \mu}\right\rangle_{\mu, 0}\right.\right. \\
& \left.+<F_{j} \alpha_{n, \mu}>_{\mu, 0}+\mu^{-1}<\alpha_{n, \mu}>_{\mu, 1}\right\},
\end{aligned}
$$

qui entraîne avec le Lemme 2.1

$$
1 \leqq \text { const. }\left\{|w(\mu / n)|+(\mu / n)^{\frac{1}{2}}+(n / \mu)^{m-l} n^{\frac{1}{2}} \mu^{-1}\right\} .
$$

On ici pose $\mu=n^{\delta}, 1>\delta>(m-l+1 / 2) /(m-l+1)$. Alors puisque tend vers zéro quand $n \rightarrow \infty$, (4.5) et (4.13) ne sont pas compatibles pour $n$ assez grand. Le théorème est ainsi démonstré.

\section{§5. Systèmes d'Orodre 2 à Coeflicients Amalytiques}

Dans ce paragraphe, nous traiterons le problème mixte pour les sysèmes d'ordre 2 à coefficients anaytiques.

Nous supposons que $A_{j}(x, t)$ soient les matrices d'ordre 2, et que leurs éléments soient analytiques. De plus, supposons que $P\left(x^{\prime}, t\right)$ soit analytique, et $A_{k}(x, t)$ et $P\left(x^{\prime}, t\right)$ aient les formes (1.6), c'est-à-dire,

$$
\begin{gathered}
A(x, t)=\left(\begin{array}{cc}
a_{1}(x, t) & 0 \\
0 & a_{2}(x, t)
\end{array}\right), a_{1}<0 \text { et, } a_{2}>0, \text { et } \\
P\left(x^{\prime}, t\right)=\left(b_{1}\left(x^{\prime}, t\right), b_{2}\left(x^{\prime}, t\right)\right), b_{1}(0,0) \neq 0 .
\end{gathered}
$$

Notre but de ce paragraph est de prouver le Théorème 1.5 sous les hypothèses ci-dessus. Nous utiliserons la méthode de Lax [3].

Démonstration $d u$ Théorème 1.5. Nous allons établir ce théorème par contradiction. Supposons que la fonction scalaire $P N^{-}$soit nul le pour $\left(x^{\prime}, t\right)=(0,0)$ et $(\lambda, \eta)=\left(\lambda_{0}, \eta^{0}\right), \operatorname{Re} \lambda_{0}>0$. Alors on va construire une solution de (1.4), qui ne satisfait pas à (1.5) dans un voisinage de l'origine. Posons la fonction cherchée $u(x, t)$ sous la forme, 


$$
u(x, t)=e^{l(x, t) n} \sum_{j=0}^{N} \frac{u_{j}(x, t)}{n^{j}},
$$

$n$ étant un entier positif, et $N$ assez grand.

Faisons opérer $\left(\frac{\partial}{\partial x_{k}}-M\right)$ à (5.3). On a

$$
\begin{aligned}
\left(\frac{\partial}{\partial x_{k}}-M\right) u & =n\left(l_{x_{k}}-M\left(l_{t}, l_{x^{\prime}}\right)\right) e^{n l} u_{0} \\
& +\sum_{j=0}^{N-1} \frac{1}{n^{j}}\left\{\left(l_{x_{k}}-M\left(l_{t}, l_{y}\right)\right) u_{j+1}+\left(\frac{\partial}{\partial x_{k}}-M\right) u_{j}\right\} e^{n l} \\
& +\frac{\left(\frac{\partial}{\partial x_{k}}-M\right) u_{N}}{n^{N}} e^{n l}
\end{aligned}
$$

où $M\left(l_{t}, l_{y}\right)=A_{k}^{-1}\left(l_{t}-\sum_{j=1}^{k-1} A_{j} l_{x_{j}}\right)$. Pour définir $l(x, t)$ et $u_{0}(x, t)$, on considère la propriété de la matrice $M(x, t ; \lambda, i \eta)$ dans un voisinage de $\left(0,0, \lambda_{0}, \eta^{0}\right)$. Puisque il découle de l'hyperbolicité que, pour $\operatorname{Re} \lambda>0$, la partie réelle du valeur propre de $M(x, t ; \lambda, i \eta)$ ne s'annule jamais, et $A_{k}$ a une valeur propre positive et une valeur propre négative, $M(x, t ; \lambda, i \eta)$ a une valeur propre à partie réelle positive, $\xi^{+}(x, t ; \lambda, i \eta)$, et une valeur propre à partie réelle négative, $\xi^{+}(x, t ; \lambda, i \eta)$. Notons que $\xi^{ \pm}(x, t ; \lambda, i \eta)$ est analytique en $(x, t, \lambda, \eta), \operatorname{Re} \lambda>0$. On détermine $l(x, t)$ par l'équation

$$
\begin{aligned}
& l_{x_{k}}=\xi^{-}\left(x, t, l_{t}, l_{x^{\prime}}\right), \text { et } \\
& l_{x_{x_{k}=0}}=l_{0}\left(x^{\prime}, t\right),
\end{aligned}
$$

où $l_{0}\left(x^{\prime}, t\right)$ est la condition initiale, qui sera déterminée dans la suite. Soit $v_{0}$ (resp. $w_{0}$ ) un vecteur-nul à droite (resp. à gauche) de $\left(l_{x_{k}}-M\left(l_{t}, l_{x^{\prime}}\right)\right.$ ). On pose

$$
u_{0}=\sigma^{(0)} v_{0}
$$

où $\sigma^{(0)}$ est la fonction scalaire. Alors, $u_{0}$ vérifie

$$
\left(l_{x_{k}}-M\left(l_{t}, l_{x^{\prime}}\right)\right) u_{0}=0
$$

Pour faire (5.4) annuler pour $j=0$, il est nécessaire que $u_{1}$ et $n_{0}$ soient vérifiés 


$$
\left(l_{x_{k}}-M\left(l_{t}, l_{x^{\prime}}\right)\right) u_{1}+\left(\frac{\partial}{\partial x_{k}}-M\right) u_{0}=0
$$

Puisque $\left(l_{x_{k}}-M\left(l_{t}, l_{x^{\prime}}\right)\right)$ est la matrice singulière, (5.9) a une solution, si le terme non-homogène $\left(\frac{\partial}{\partial x_{k}}-M\right) u_{0}$ est orthogonal au vecteur-nul à droite $w_{0}$ de $\left(l_{x_{k}}-M\left(l_{t}, l_{x^{\prime}}\right)\right)$, c'est-à-dire,

$$
\begin{aligned}
& w_{0} \cdot\left(\frac{\partial}{\partial x_{k}}-M\right) u_{0} \\
& =\sigma_{x_{k}}^{(0)}-b_{k} \sigma_{t}^{(0)}+\sum_{j=1}^{k-1} b_{j} \sigma_{x_{j}}^{(0)}+b \sigma^{(0)}=0,
\end{aligned}
$$

où $a_{1}=w_{0} \cdot A{ }^{1} v_{0}, b_{j}=w_{0} \cdot A^{-1} A_{j} v_{0}$ et $b=w_{0} \cdot\left(\frac{\partial}{\partial x_{k}}-M\right) v_{0}$, et ici on a utilisé $w_{0} \cdot v_{0}=1$. Soit $u_{1}^{(0)}$ une solution particulière de (5.9).

On pose $u_{1}=u_{1}^{(0)}+\sigma^{(1)} v_{0}$. Alors $u_{1}$ et $u_{0}$ satisfont à (5.9).

De proche en proche, en posant au zéro les termes de la même puissance de $n$ dans (5.4), on a les formes suivantes

$$
\left(l_{x_{k}}-M\left(l_{t}, l_{x^{\prime}}\right)\right) u_{j}+\left(\frac{\partial}{\partial x_{k}}-M\right) u_{j-1}=0
$$

pour $j=1,1,2, \ldots, N$. Alors, de la même manière que $u_{1}$ on peut construire $u_{j}$ tel que

$$
u_{j}=u_{j}^{(0)}+\sigma^{(j)} v_{0}
$$

ici $\sigma^{(j)}, j=1, \ldots, N$ vérifient

$$
\sigma_{x_{k}}^{(j)}-b_{k} \sigma_{t}^{(j)}+\sum_{s=1}^{k-1} b_{s} \sigma_{x_{s}}^{(j)}=-w_{0} \cdot\left(\frac{\partial}{\partial x_{k}}-M\right) u_{j}^{(0)}
$$

où $u_{j}^{(0)}$ étant des solutions particulières de (5.11).

On peut chercher les solutions des équations (5.5), (5.9) et (5.13), en utilisant le théorème de Cauchy-Kowalevski.

Nous allons maintenant déterminer les conditions initiales telles que

$$
\left.P u_{j}\right|_{x_{k}=0}=0 \text {, pour } j=0,1, \ldots, N \text { 。 }
$$

Pour cela, nous considérons $P N^{-}$au voisinage de $\left(0,0 ; \lambda_{0}, \eta^{0}\right)$. Puisque les racines caractéristiques de $M(x, t ; \lambda, i \eta)$ sont simples au voisinage de 
$\left(0,0, \lambda_{0}, \eta^{0}\right)$, on a

$$
N^{-1} M N=\left(\begin{array}{cc}
\xi^{+} & 0 \\
0 & \xi^{-}
\end{array}\right)
$$

Donc on peut poser $N^{-}=v_{0}$. Pour simplifier, on écrit $r\left(x^{\prime}, t ; \lambda, i \eta\right)=$ $\mathbb{P}\left(x^{\prime}, t\right) v_{0}\left(x^{\prime}, 0, t ; \lambda, i \eta\right)$. Alors, de l'hypothèse il décole que $r\left(0,0, \lambda_{0}\right.$, $\left.i \eta^{0}\right)$ soit zéro.

Lemme 5.I.Pour $\left(\lambda_{0}, \eta^{0}\right)$ tel que $r\left(0,0 ; \lambda_{0}, i \eta^{0}\right)=0$, on $a$ $\left(\frac{\partial}{\partial \lambda} r\right)\left(0,0 ; \lambda_{0}, i \eta^{0}\right) \neq 0$.

Preuve. Pour simplifier la notation, on écrit $P_{0}=P(0,0), v_{0}(\lambda)=$ $v_{0}\left(0,0 ; \lambda, i \eta^{0}\right)$ et $r_{0}(\lambda)=r\left(0,0 ; \lambda, i \eta^{0}\right)$. On a l'égalité $r_{0}(\lambda)=P_{0} v_{0}(\lambda)$. Puisque $v_{0}(\lambda)$ est le vecteur propre correspondant à la valeur propre $\xi_{0}^{-}(\lambda)$ $=\xi^{-}\left(0,0 ; \lambda, i \eta^{0}\right)$ de $M_{0}(\lambda)=M\left(0,0 ; \lambda, i \eta^{0}\right)$, (5.15) se récrit

$$
M_{0}(\lambda) v_{0}(\lambda)=\xi_{0}^{-}(\lambda) v_{0}(\lambda)
$$

En le dérivant par repport à $\lambda$, on a

$$
A_{k}^{-1}(0,0) v_{0}(\lambda)+M_{0}(\lambda) v_{0}(\lambda)=\xi_{0 \lambda}^{-}(\lambda) v_{0}(\lambda)+\xi_{0}^{-}(\lambda) v_{0 \lambda}(\lambda)
$$

Si $r_{0 \lambda}\left(\lambda_{0}\right)=0$, d'après $r_{0 \lambda}\left(\lambda_{0}\right)=P_{0} v_{0 \lambda}\left(\lambda_{0}\right)$, on a $v_{0 \lambda}\left(\lambda_{0}\right)=\alpha v_{0}\left(\lambda_{0}\right)$, où $\alpha$ est un scalaire. Donc, compte tenu de $(5.16)$, on a la relation $A^{-1}(0,0) v_{0}\left(\lambda_{0}\right)$ $=\xi_{0}^{-}\left(\lambda_{0}\right) v_{0}\left(\lambda_{0}\right)$. Donc $v_{0}\left(\lambda_{0}\right)$ est le vecteur propre et $\xi_{0}^{-}\left(\lambda_{0}\right)$ est la valeur propre de $A^{-1}(0,0)$. De la forme $(5.1)$ de $A_{k}(0,0)$ il découle que $v_{0}\left(\lambda_{0}\right)$ ${ }^{t}(1,0)$. D'autre part, $r_{0}\left(\lambda_{0}\right)=P_{0} v_{0}\left(\lambda_{0}\right)=b_{1}(0,0)$ ne s'annule pas de l'hypothèse (5.2). Cela implique une contradiction.

Il découle du Lemme 5.1 que l'on a une fonction $\lambda\left(x^{\prime}, t ; i \eta\right)$ analytique en $\left(x^{\prime}, t, \eta\right)$ au voisine de $\left(0,0, \eta^{0}\right)$ telle que

$$
r\left(x^{\prime}, t ; \lambda\left(x^{\prime}, t ; i \eta\right), i \eta\right)=0
$$

Maintenant on va déterminer la condition initiale de $l(x, t)$, solution de (5.5). On choisit la condition initiale $l_{0}\left(x^{\prime}, t\right)$, qui vérifie l'équation suivante 


$$
\begin{aligned}
& l_{0 t}\left(x^{\prime}, t\right)=\lambda\left(x^{\prime}, t, l_{0 x^{\prime}}\right) \\
& l_{0}\left(x^{\prime}, 0\right)=i x^{\prime} \cdot \eta^{0}
\end{aligned}
$$

Du théorème Cauchy-Kowalevski il découle qu'il existe une solution de (5.18). Alors on a d'après (5.17) et (5.18),

$$
\begin{aligned}
\left.P v_{0}\right|_{x_{k}=0} & =r\left(x^{\prime}, t, l_{0 t}, l_{0 x^{\prime}}\right), \\
& =0 .
\end{aligned}
$$

Dans la suite, nous allons déterminer la condition initiale de $\sigma^{(j)}$ telle que

$$
\left.u_{j}^{(0)}\right|_{x_{k}=0}=0,(j=1,2, \ldots, N),
$$

où $u_{j}^{(0)}$ étant la solution particulière de (5.11). Pour cela, il faut et it suffit que pour $w_{1}(x, t)$ le vecteur-nul à gauche de $\left(\xi^{+}\left(x, t, l_{t}, l_{x^{\prime}}\right)-M\left(l_{t}\right.\right.$, $\left.l_{x^{\prime}}\right)$ ), on ait

$$
\left.w_{1} \cdot\left(\frac{\partial}{\partial x_{k}}-M\right) u_{j-1}\right|_{x_{k}=0}=0
$$

ou en posant $\sigma^{(j-1)}\left(x^{\prime}, 0, t\right)=\sigma_{0}^{(j-1)}\left(x^{\prime}, t\right)$, on récrit

$$
d_{k} \sigma_{0 t}^{(j-1)}+\sum_{j=1}^{k-1} d_{j} \sigma_{0 x_{j}}^{(j-1)}+d \sigma_{0}^{(j-1)}=0
$$

ici, $d_{k}=w_{1} \cdot A_{k}^{-1} v_{0}, d_{j}=w_{1} \cdot A_{k}^{-1} A_{j} v_{0}$ et $d=w_{1} \cdot\left(\frac{\partial}{\partial x_{k}}-M\right) v_{0}$ et on a utilisé $w_{1} \cdot v_{0}=0$. Notons qu'il découle de $w_{1} \cdot v_{0}=0$ et de la forme de $A_{k}$ que $d_{k}$ ne s'annule pas. Donc, d'après le théorème de Cauchy-Kowalevski, on peut trouver la solution de (5.21).

Maintenant nous allons montrer que la fonction (5.3), dont nous avons construit, ne satisfait pas à (1.5). On pose

$$
\rho=\max _{(x, t) \in \bar{\Omega}} \operatorname{Re} l(x, t)=\operatorname{Re} l\left(x_{0}, t_{0}\right) .
$$

Notons qu'il découle du primier terme de (5.18) que la partie réelle de $l_{t}(x, t)$ est positive dans $\Omega$. Donc on a

$$
\rho>\rho_{0}=\max _{x \in \bar{D}} \operatorname{Re} l(x, 0)
$$


La fonction $u(x, t)$ définie par (5.3) vérifie

$$
\left(\frac{\partial}{\partial x_{k}}-M\right) u=\frac{1}{n^{N}}\left(\frac{\partial}{\partial x_{k}}-M\right) u_{N} e^{n l} \equiv f
$$

Donc on a

$$
|f|_{s, \Omega} \leqq \text { const. } \frac{1}{n^{N-s}} e^{n_{\rho}}
$$

De plus, la valeur initiale $u(x, 0)(=g(x))$ de $u(x, t)$ satisfait à

$$
|g|_{s, D} \leqq \text { const. } n^{s} e^{\rho_{0}{ }^{n}} \text {. }
$$

En vertu de (5.19) et de (5.20), on a

$$
\left.P u\right|_{x_{k}=0}=0 \text {. }
$$

D'autre part, d'après (5.22) on a

$$
\begin{aligned}
\left|u\left(x_{0}, t_{0}\right)\right| & \geqq e^{\rho n}\left(\left|u_{0}\left(x_{0}, t_{0}\right)\right|-O\left(\frac{1}{n}\right)\right), \\
& \geqq \text { const. } e^{\rho n} .
\end{aligned}
$$

Donc, il découle de (5.23) que $u(x, t)$ ne satisfait pas à (1.5) pour $n$ assez grand et $N=s+1$. Cela prouve le Théorème 1.5 .

\section{References}

[1] Agemi, R. and Shirota, T., On necessary and sufficient conditions for $L^{2}$-wellposedness of mixed problems for hyperbolic equations II. Jour. Fac. Sci. Hokaido Univ., 22, (1972), 137-149.

[2] Hersh, R., Mixed problem in several variables, J. Math. Mech. 12 (1963), 317-334.

[3] , Boundary conditions for equations of evolution, Arch. Rational Mech. Anal. 16 (1964) 243-264.

[4] Kreiss, O.K., Initial boundary value problems for hyperbolic systems, Comm. Pure Appl. Math. 23 (1970), 277-298.

[5] Kajitani, K., A necessary condition for the $L^{2}$-well posed Cauchy problem with variable coefficients, J. Math. Kyoto Univ. 13 (1973), 701-711.

[6] Lax, P. D., Asymtotic solutions of oscillatory intial value problems, Duke Math. J. 24 (1957), 627-646.

[7] Sakamoto, R., $L^{2}$-well-posedeness for hyperbolic mixed problems, à paraitre. 